\title{
Evaluación del uso de los humedales de manglar como biofiltro de efluentes de camaroneras en Yucatán, México
}

\author{
Evaluation of use mangrove wetland as a biofilter of \\ shrimp pond effluent in Yucatan, Mexico
}

\section{Arturo Zaldívar-Jiménez ${ }^{1}$, Jorge Herrera-Silveira ${ }^{1}$, Rosela Pérez-Ceballos ${ }^{1}$ y Claudia Teutli-Hernández ${ }^{1}$}

${ }^{1}$ Centro de Investigación y de Estudios Avanzados, Instituto Politécnico Nacional, Unidad Mérida, Antigua Carreta a Progreso Km. 6, Apartado Postal 73, Cordemex, 97310, Mérida, Yucatán, México. arturozj@gmail.com

\begin{abstract}
An area of mangrove on the coast of Yucatan (Mexico) was used as biofilter for the discharge of effluents from a shrimp farm intensive cultivation during 5 years. In order to assess the functional capacity of mangroves to reduce nutrient loading and suspended matter in the effluent, water quality variable as $\mathrm{NO}_{2}{ }^{-}+\mathrm{NO}_{3}{ }^{-}, \mathrm{NH}_{4}{ }^{+}, \mathrm{PO}_{4}{ }^{-3}$, total suspended solids (TSS), volatile suspended solids (VSS) and chlorophyll-a were determined. Sampling stations were established in the influent, effluent, biofilter and reference sites in both the marsh and in the sea area adjacent to the farm, sampling was monthly. Also the ponds were sampled biweekly to determine the production and loss of nutrients through a mass balance. The water quality results indicated that the $\mathrm{NO}_{2}{ }_{2}+\mathrm{NO}_{3}{ }^{-}$concentrations in the influent was higher with $4.69 \mathrm{mg} \mathrm{l}^{-1}$ compared to $0.56 \mathrm{mg} \mathrm{l}^{-1}$ registered in the effluent. The $\mathrm{NH}_{4}^{+}$was $0.11 \mathrm{mg} \mathrm{l}^{-1}$ and $0.56 \mathrm{mg} \mathrm{l}^{-1}$ in the influent and effluent, respectively. The $\mathrm{PO}_{4}^{-3}$ was higher in the effluent with $0.18 \mathrm{mg} \mathrm{l}^{-1}$ while in the influent was $0.06 \mathrm{mg} \mathrm{l}^{-1}$. TSS, VSS and chlorophyll-a increased its concentrations in the effluent from the ponds. According to mass balance effluents exported $359 \mathrm{~kg} \mathrm{ha}^{-1} \mathrm{yr}^{-1}$ and $42 \mathrm{~kg}^{\circ}$ $\mathrm{ha}^{-1} \mathrm{yr}^{-1}$ of $\mathrm{N}$ and $\mathrm{P}$, respectively. However, despite the load of nutrients and suspended solids in the discharge of the farm, the biofilter mangrove had the ability to retain $44 \%$ of the $\mathrm{NO}_{2}{ }^{-}+\mathrm{NO}_{3}, 55 \%$ of $\mathrm{NH}_{4}{ }^{+}$and $80 \%$ of $\mathrm{PO}_{4}^{-3}$ after the effluent passed through mangrove biofilter. This study demonstrates the efficiency of mangroves in retaining nutrients, so that could be considered as an alternative for the treatment of effluents from shrimp farms.
\end{abstract}

Key words: Mangroves, biofilter, wetlands, shrimp farm, effluents

Resumen.- Un área de manglar en la costa de Yucatán (México) fue utilizada como biofiltro durante la descarga de efluentes de una granja camaronera de cultivo intensivo que ha funcionado durante 5 años. Con el propósito de evaluar la capacidad funcional del manglar para reducir la carga de nutrientes y materia suspendida de los efluentes, se determinaron variables indicadoras de la calidad de agua como $\mathrm{NO}_{2}{ }^{-}+\mathrm{NO}_{3}, \mathrm{NH}_{4}{ }^{+}, \mathrm{PO}_{4}^{-3}$, sólidos suspendidos totales (SST), sólidos suspendidos volátiles (SSV) y clorofila-a. Se establecieron estaciones de muestreos en los influentes, efluentes, biofiltro y sitios de referencia tanto en la ciénaga como en la zona marina adyacente a la granja; los muestreos fueron mensuales. También se realizaron muestreos quincenales en los estanques de cultivo para determinar la producción y pérdidas de los nutrientes a través de un balance de masas. Los resultados de calidad del agua indicaron que la concentración de $\mathrm{NO}_{2}+\mathrm{NO}_{3}$ en el influente fue mayor con 4,69 $\mathrm{mg} \mathrm{l}^{-1}$ en comparación con $0,56 \mathrm{mg} \mathrm{l}^{-1}$ registrada en el efluente. $\mathrm{El} \mathrm{NH}_{4}^{+}$fue $0,11 \mathrm{mg} \mathrm{l}^{-1} \mathrm{y}^{0,56} \mathrm{mg} \mathrm{l}^{-1}$ en el influente y efluente respectivamente. $\mathrm{El} \mathrm{PO}_{4}^{-3}$ fue mayor en los efluentes con 0,18 $\mathrm{mg} \mathrm{l}^{-1}$ que en el influente con 0,06 $\mathrm{mg} \mathrm{l}^{-1}$. Los SST, SSV y clorofila-a incrementaron su concentración en los efluentes de los estanques. De acuerdo al balance de masas, con los efluentes se exportaron $359 \mathrm{~kg} \mathrm{ha}^{-1} \mathrm{año}^{-1}$ y $42 \mathrm{~kg} \mathrm{ha}^{-1} \mathrm{año}^{-1}$ de N y P, respectivamente. Sin embargo, a pesar de la carga de nutrientes y sólidos suspendidos en la descarga de la granja, el biofiltro de manglar presentó la capacidad de retener el $44 \%$ del $\mathrm{NO}_{2}{ }^{-}+\mathrm{NO}_{3}$; el $55 \%$ del $\mathrm{NH}_{4}{ }^{+}$y el $80 \%$ del $\mathrm{PO}_{4}^{-3}$ después de que los efluentes pasaron por el biofiltro de manglar. Este estudio demuestra la eficiencia de los manglares para retener nutrientes, por lo que podrían ser considerados como una alternativa para el tratamiento de efluentes de camaroneras.

Palabras clave: Manglares, biofiltro, humedales, camaroneras, efluentes 


\section{INTRODUCCIÓN}

Diferentes investigaciones han reconocido la capacidad de los humedales naturales y construidos para remover nutrientes y contaminantes del agua, por lo que han sido considerados para el tratamiento de aguas residuales (Nedwell 1975, Kadlec \& Knight 1996, Tilley et al. 2002, Primavera et al. 2007, Wu et al. 2008). En todos los humedales incluyendo a los manglares, la filtración, fijación física, precipitación química, absorción, adsorción, procesos biológicos, transformación y almacenamiento por vegetación y desnitrificación, son características funcionales que permiten a los manglares ser eficaces en la remoción de nutrientes y otros materiales (Twilley \& Rivera-Monroy 2009). Por estas razones, se valoran a los humedales como sistemas naturales que brindan el servicio ecológico de mantener la calidad de agua en los ambientes acuáticos a los cuales se encuentra asociados (Adame et al. 2010). Los manglares remueven nutrientes disueltos en el agua, funcionan como sumidero de nitrógeno (Rivera-Monroy \& Twilley 1996) y fósforo (Alongi 1996). Sin embargo, a pesar del papel que pueden tener estos sistemas para mantener o mejorar la calidad del agua, existen necesidades de información sobre los procesos químicos y físicos que intervienen en la retención y transformación de los materiales que ingresan al manglar. El uso de los manglares como biofiltro representa una alternativa para mitigar la degradación de los ecosistemas de manglar a través de la restauración ecológica y manejo integrado del sistema camaroneramanglar.

Es reconocido que los efectos en el suelo del manglar en el corto plazo están dados por el incremento en la disponibilidad de nutrientes para la planta; pero, los efectos a largo plazo de la fertilización todavía son poco claros y que podrían ser negativos sin ningún manejo (Wong et al. 1995, Trott \& Alongi 2000, McKee et al. 2007). Otros autores señalan que el enriquecimiento con nutrientes puede incrementar la sensibilidad del manglar a la hipersalinidad (Lovelock et al. 2009), el incremento de la herbivoría y depredación por escarabajos barrenadores (Feller \& Chamberlain 2007).

Una práctica común de las camaroneras es la descarga de los efluentes directamente al mar, estuarios o lagunas, debido al arraigado convencimiento de la capacidad del mar y de los ecosistemas costeros para amortiguar y diluir los contaminantes (Sánchez-Carrillo \& Alvarez-Yépiz 2008). De hecho, en Yucatán, Golfo de México, los impactos ambientales producidos por la actividad camaronera han estado principalmente asociados a convertir áreas de manglar y duna costera por áreas de estanques para el cultivo de camarón (CONABIO 2008, Olvera-Novoa 2010). Además, los efectos negativos han impactado en la calidad del agua por descargar los efluentes a las aguas costeras. Reyes (2001) cuantificó que las descargas de efluentes de camaroneras a la zona marina de Yucatán pueden ser hasta de $54 \mathrm{~kg} \mathrm{ha}^{-1}$ para nitrógeno inorgánico disuelto y $10 \mathrm{~kg} \mathrm{ha}^{-1}$ para fósforo reactivo soluble. Por otra parte, en la zona marina adyacente a la granja camaronera, Morales-Ojeda \& Herrera-Silveira (2010) atribuyeron al incremento en la columna de agua la concentración del nitrógeno y fósforo inorgánico por efecto de largo plazo de las descarga de efluentes de manera directa al mar en Yucatán.

En este estudio, un biofiltro constituido por manglares fue usado como receptor de los efluentes de una granja camaronera de cultivo intensivo. Este biofiltro permitió evaluar la viabilidad potencial del uso del manglar para el tratamiento de efluentes a través de los cambios en las características de la calidad del agua de los efluentes. Para ello fue necesario caracterizar la calidad de agua a través de los componentes físicos y químicos de los efluentes de la camaronera y su comparación con sistemas naturales; conocer los valores en la producción de nitrógeno y fósforo en relación con el ciclo de cultivo del camarón, a través de un análisis de balance de masas de los nutrientes en los estanques; y por ultimo, determinar las diferencias de la carga de nutrientes y materiales suspendidos en los efluentes antes y después de pasar por un biofiltro de manglar, para establecer la eficiencia del biofiltro y potencial uso de este ecosistema para el tratamiento de efluentes de camaroneras.

\section{Materiales y Métodos}

\section{DESCRIPCIÓN AMBIENTAL DEL SITIO}

La granja camaronera se localiza en Puerto Sisal en la costa noroeste de la Península de Yucatán, México (21ํํำ $10^{\prime} 24,44^{\prime \prime} \mathrm{N}, 89^{\circ} 59^{\prime} 52,18^{\prime \prime O}$ ). En esta localidad las lluvias son poco frecuentes, el clima es semiárido con una estación de sequía bien establecida entre marzo-junio, una estación de lluvias de julio-octubre y una estación con la llegada de frentes polares de noviembre-febrero. La evaporación promedio en los últimos 50 años fue de $1818 \mathrm{~mm}_{\text {año }}{ }^{-1} \mathrm{y}$ de $513 \mathrm{~mm}$ año-1 ${ }^{-1}$ para la precipitación; la temperatura media fue de $26,57^{\circ} \mathrm{C}$ (CNA 2010). El biofiltro colinda con la ciénaga, y esta se caracteriza por presentar vegetación de manglar a manera de islotes, es un cuerpo de agua somero $(0,20$ a $0,45 \mathrm{~m})$ que depende de la precipitación y de las 
descargas de agua subterránea (Herrera-Silveira \& RamírezRamírez 1997). En la ciénaga se presenta la degradación de los manglares debido a cambios en el hidroperíodo por la apertura de bocanas que conectaron la cienega con el mar, por lo que existen necesidades de restauración (ZaldívarJiménez et al. 2001).

\section{DESCRIPCiÓN DE LA GRANJA DE CAMARÓN}

La granja camaronera Industrias Pecis S.A., Yucatán, México, tenía la capacidad de producir 2000 ton de camarón blanco (Litopenaeus vannammei) al año en 80 ha de estanques. La fuente principal de agua (influente) para los estanques provenía de la extracción de agua salobre del subsuelo. La tasa de recambio diario de agua fue en promedio del $10 \%$ y en un tiempo total por ciclo de cultivo de 105 días. Del total de estanques de Industrias Pecis, 14 de ellos con dimensión de 2,5 ha (35 ha en estanques) descargaban sus efluentes en el biofiltro de manglar con un área de 13,17 ha (Fig. 1). La práctica de descargar efluentes al biofiltro se llevó a cabo durante 5 años (2002-2007); los demás estanques (45 ha en total) descargaban sus efluentes directamente al mar y esto fue durante 10 años (1997-2007). El biofiltro estuvo constituido por un área lagunar de 9,20 ha con profundidad media de $0,7 \mathrm{~m}$ y un manglar tipo cuenca dominado por Avicennia germinans de 3,97 ha. Los árboles de $A$. germinans tuvieron en altura promedio 2,90 m y densidad de 3441 árbol haํ․ El tiempo máximo de renovación del agua en el biofiltro fue estimado en 6,9 días, que fue el tiempo necesario para el total recambio de agua de un volumen conocido del biofiltro (Kadlec \& Knight 1996). Tanto los estanques como el biofiltro estaban delimitados por bordos artificiales con materiales de la región. El suelo del biofiltro estuvo compuesto de material orgánico (restos de manglar y del proceso de producción del camarón) e inorgánico (limos y arenas). La densidad del suelo fue de $0,22 \mathrm{~g} \mathrm{~cm}^{-3}$ y materia orgánica de $22 \%$ en los primeros $5 \mathrm{~cm}$ de la superficie; después de los $10 \mathrm{~cm}$ de profundidad del suelo la densidad se incrementa a $0,87 \mathrm{~g} \mathrm{~cm}^{-3}$ y disminuye el contenido de materia orgánica a 7\%.

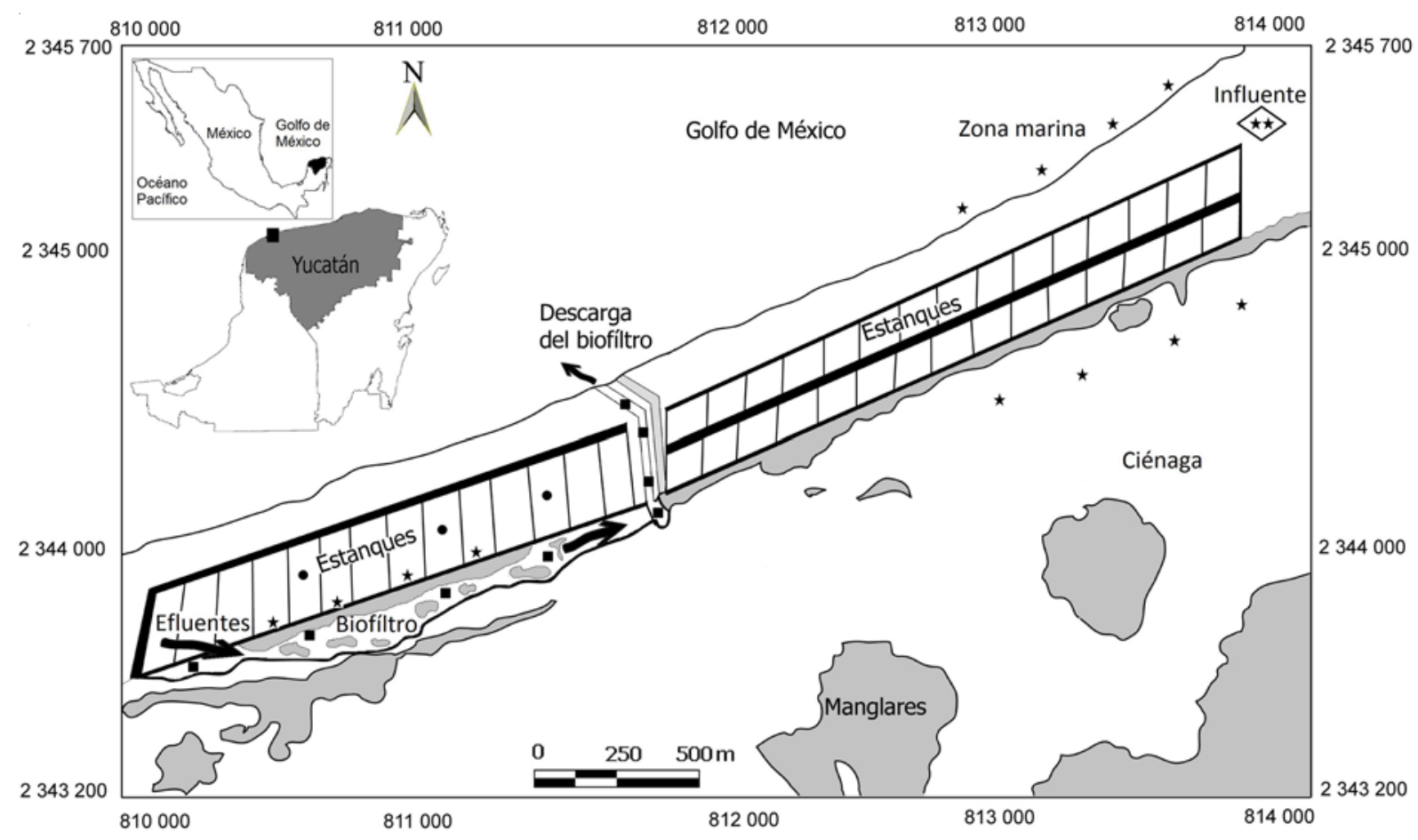

Figura 1. Localización de la granja camaronera Pecis en la costa norte de la Península de Yucatán. Las flechas indican la dirección de flujo de los efluentes. Identificación de estaciones: caracterización de la calidad de agua $(\star)$, producción e intercambio de nutrientes $(\bullet)$ y determinación de la eficiencia del biofiltro ( $\boldsymbol{\nabla})$ / Location of Pecis shrimp farm at the northern coast of Yucatan Peninsula. The arrows indicate the direction of flow of effluent. Station ID: water quality characterization ( $\star$ ), production and exchange of nutrients $(\bullet)$ and determination of efficiency of the biofilter 
El manejo de los efluentes consistía en que descargaban de los estanques en forma directa y por gravedad escurrían al biofiltro, de este descargaba por gravedad, a través de un canal artificial al mar (Fig. 1).

\section{Análisis de la calidad de AgUa}

Para la caracterización de la calidad de agua se establecieron 2 estaciones de muestreo en el influente y 4 en los efluentes que descargaban en el biofiltro, también se establecieron estaciones de muestreo en sitios de referencia como fue la ciénaga y la zona marina adyacentes a la granja con 4 estaciones cada una (Fig. 1). Se tomaron muestras mensuales de agua para determinar las variables químicas entre febrero y julio de 2006. En cada estación se tomaron datos por duplicado in situ con una sonda multiparámetros modelo YSI 6600 de temperatura $\left({ }^{\circ} \mathrm{C}\right)$, salinidad y oxígeno disuelto $\left(\mathrm{mg} \mathrm{l}^{-1}\right)$. Se tomaron muestras por duplicado de agua en cada estación para la determinación de nutrientes inorgánicos disueltos, sólidos suspendidos totales ( $\mathrm{SST}, \mathrm{mg} \mathrm{l}^{-1}$ ) y sólidos suspendidos volátiles (SSV, $\mathrm{mg} \mathrm{l}^{-1}$ ). En el caso de los nutrientes inorgánicos disueltos, el fósforo reactivo soluble $\left(\mathrm{PO}_{4}^{-3}, \mathrm{mg} \mathrm{l}^{-1}\right)$ se determinó de acuerdo con Murphy \& Riley (1962) con las modificaciones de Strickland \& Parsons (1972). El amonio $\left(\mathrm{NH}_{4}^{+}, \mathrm{mg} \mathrm{l}^{-1}\right)$ por el método del fenol-hipoclorito (Solorzano 1969). El nitrógeno como nitrito $\left(\mathrm{NO}_{2}^{-}, \mathrm{mg} \mathrm{l}^{-1}\right)$ por la técnica de la sulfanilamida en medio ácido y los nitratos $\left(\mathrm{NO}_{3}^{-}, \mathrm{mg} \mathrm{l}^{-1}\right)$ se determinaron como $\mathrm{NO}_{2}{ }^{-}$después de pasar la muestra a través de una columna de $\mathrm{Cd}-\mathrm{Cu}$ (Parsons et al. 1984). La clorofila- $a\left(\mu \mathrm{g} \mathrm{l}^{-1}\right)$ se cuantificó por espectrofotometría mediante la extracción de acetona al $90 \%$, las mediciones se realizaron a $664 \mathrm{~nm}$ y $647 \mathrm{~nm}$ y fue calculada de acuerdo a la fórmula de Jeffrey \& Humphrey (Parsons et al. 1984). Los iones nitrogenados $\left(\mathrm{NO}_{2}^{-}, \mathrm{NO}_{3}^{-}, \mathrm{NH}_{4}^{+}\right)$se sumaron para cuantificar el nitrógeno inorgánico disuelto (NID).

Dentro de los estanques de cultivo se realizó un análisis de balances de masas del nitrógeno y fósforo mediante el seguimiento de la calidad de agua en 3 estanques durante un ciclo de producción (desde la siembra hasta la cosecha de camarones). Durante 105 días que duró el ciclo de producción, en cada estanque se establecieron 3 estaciones de muestreo, además se tomaron muestras en el influente y efluente por duplicado. Los muestreos fueron realizados en intervalos de tiempo quincenales desde marzo a junio. Las variables analizadas fueron las ya descritas. Con este diseño de muestreo se determinó la producción de nutrientes y balance de masas entre entradas y salidas de nitrógeno y el fósforo en cada estanque; ya que en el caso de las salidas, la concentración de nutrientes y sólidos suspendidos de los efluentes dependen de la etapa de cultivo y procesos que ocurren dentro de los estanques de camarón. Las vías de entrada de nutrientes $\left(\mathrm{kg} \mathrm{ha}^{-1} \mathrm{año}^{-1}\right)$ a los estanques de cultivo fueron: contenido en larvas de camarones, influente, fertilizantes, alimentos; mientras que las vías de salida fueron: contenido en camarones cosechados, efluentes, sedimentación y otras vías que no fueron medidas y contabilizadas pero que constituyen un remanente. El remanente se calculó mediante la resta del total de las vías de entradas menos las vías de salida de la cosecha, los efluentes y los sedimentos. El volumen total de agua de recambio por cada hectárea de estanque por año $\left(\mathrm{m}^{3} \mathrm{ha}^{-1}\right.$ año $\left.{ }^{-1}\right)$ fue estimado según las especificaciones de Nath \& Bolte (1998).

En el biofiltro se establecieron estaciones de muestreo para comparar la eficiencia de este sobre la condición de los efluentes. También se hicieron mediciones en la entrada del biofiltro (efluentes) y en la salida (descargas del biofiltro). En todos los casos se establecieron 4 estaciones para la toma de muestras por duplicado. Los muestreos fueron mensuales entre febrero y julio. Se midieron los flujos de agua $\left(\mathrm{m}^{3}\right.$ día $\left.^{-1}\right)$ en las entradas del biofiltro (efluentes) y en la salida (descarga del biofiltro) durante cada muestreo según las especificaciones de Nath \& Bolte (1998), con ello se cuantificó la carga de nutrientes multiplicando el flujo por la concentración (kg día $\left.{ }^{-1}\right)$. Para el balance de agua en el biofiltro, el efecto de la precipitación con 1,24 mm día ${ }^{-1}$ y la evaporación de 4,3 $\mathrm{mm}$ día $^{-1}$ no fueron consideradas.

\section{ANÁLISIS DE DATOS}

Se empleó un análisis de varianza no paramétrico de Kruskal-Wallis (Kruskal \& Wallis 1952, Quinn \& Keough 2002) para determinar las diferencias en las características del agua (salinidad, temperatura, oxígeno disuelto, SST, SSV, $\mathrm{PO}_{4}^{-3}, \mathrm{NO}_{3}^{-}+\mathrm{NO}_{2}^{-}, \mathrm{NH}_{4}^{+}$, NID y clorofila-a) entre tratamientos (influente, efluente, zona marina y ciénaga). Cuando se detectaron diferencias significativas entre las características del agua, se realizaron comparaciones de pares de medianas a través del grafico de cajas y bigotes (Statgraphics Centurion XVI 2010). Por otra parte, también se determinaron las diferencias entre las variables de los efluentes, biofiltro y la descarga del biofiltro; por último, se comparó la calidad del agua antes y después de pasar por el biofiltro. El balance de masas en los estanques fue calculado sustrayendo las cargas totales de las vías de entrada a las vías de salida, expresados en $\mathrm{kg} \mathrm{ha}^{-1} \mathrm{año}^{-1}$. 


\section{Resultados}

\section{Caracterización dE LA CALIDAD DE AgUa}

La concentración de $\mathrm{NO}_{2}{ }^{-}+\mathrm{NO}_{3}{ }^{-}$fue significativamente mayor en el influente (4,68 $\left.\mathrm{mg} \mathrm{l}^{-1}\right)$ que en el efluente $(0,56$ $\left.\mathrm{mg} \mathrm{l}^{-1}\right)$; la concentración en los sitios de referencia fueron $0,44 \mathrm{mg} \mathrm{l}^{-1}$ en la zona marina y $0,28 \mathrm{mg} \mathrm{l}^{-1}$ en la ciénaga. La concentración de $\mathrm{NH}_{4}^{+}$fue mayor en el efluente con 0,56 $\mathrm{mg} \mathrm{l}^{-1}$ y menor con $0,11 \mathrm{mg} \mathrm{l}^{-1}$ en el influente; el amonio en el efluente fue mayor que en la ciénaga (Tabla 1).

El NID fue mayor en el influente (4,83 $\left.\mathrm{mg} \mathrm{l}^{-1}\right)$ y efluentes (1,34 $\left.\mathrm{mg} \mathrm{l}^{-1}\right)$ comparado con la concentración en la zona marina y la ciénaga. La concentración de $\mathrm{PO}_{4}^{-3}$ fue significativamente mayor $(P<0,01)$ en los efluentes con 0,18 $\mathrm{mg} \mathrm{l}^{-1}$ en comparación con el influente con 0,06 mg $\mathrm{l}^{-1}$, la ciénaga y zona marina presentaron valores menores a $0,09 \mathrm{mg} \mathrm{l}^{-1}$ (Tabla 1 ). Respecto a la temperatura y oxígeno disuelto en la columna de agua, no se identificaron diferencias ( $P>0,91$ y $P>0,54$, respectivamente); la temperatura varió entre $25,3^{\circ} \mathrm{C}$ y $27,6^{\circ} \mathrm{C}$, el oxígeno disuelto fue menor en la zona marina con 5,22 $\mathrm{mg} \mathrm{l}^{-1} \mathrm{y}$ mayor en los efluentes con 5,88 $\mathrm{mg} \mathrm{l}^{-1}$. Los SST fueron mayores en los efluentes y en la zona marina con concentraciones mayores a 163,4 $\mathrm{mg} \mathrm{l}^{-1}$. El valor medio de SSV fue mayor en los efluentes (52,0 $\mathrm{mg} \mathrm{l}^{-1}$ ) indicando alta exportación de materia orgánica comparado con la concentración promedio de 33,4 $\mathrm{mg} \mathrm{l}^{-1}$ en los sitios de referencia. La clorofila-a registró valores en el efluente de 157,1 $\mathrm{\mu g} \mathrm{l}^{-1}$ comparado con los otros tratamientos de 5,99 $\mu \mathrm{g} \mathrm{l}^{-1}$ en la ciénaga y 1,84 $\mu \mathrm{g} \mathrm{l}^{-1}$ en el agua del influente (Tabla 1).
Producción e intercambio de nUtrientes en los ESTANQUES DE CULTIVO

La entrada principal de NID al estanque fue a través del agua de influente con $983 \mathrm{~kg} \mathrm{ha}^{-1}$ año-1 contribuyendo con el 61\% de las entradas del nitrógeno. La segunda vía de entrada fue la adición de alimentos con promedio de $612 \mathrm{~kg} \mathrm{ha}^{-1}$ año ${ }^{-1}$, y que corresponde al 39\% de todas las fuentes de NID en cada ciclo de cultivo (Tabla 2). La fertilización y el contenido de nitrógeno en larvas de camarón solo contribuyeron con el 0,1\% de las entradas al estanque de cultivo. Respecto a las salidas del NID, se cuantificó que la cosecha fue la principal vía de recuperación con el $65 \%$ del total (1034 $\mathrm{kg} \mathrm{ha}^{-1}$ año-1 de nitrógeno) (Tabla 3). A través de los efluentes se exportó $359 \mathrm{~kg} \mathrm{ha}^{-1}$ año-1 de NID hacia el biofiltro (22\% de las salidas). El nitrógeno acumulado en los sedimentos del estanque al final de cada ciclo de cultivo contribuyó con $67 \mathrm{~kg} \mathrm{ha}^{-1}$ año-1 (4\% del total). De las vías de salida $138 \mathrm{~kg}$ ha $^{-1}$ año ${ }^{-1}$ (9\%) fue considerado como remanente (vías no registradas) de nitrógeno (Tabla 2 ).

Respecto al $\mathrm{PO}_{4}^{-3}$, la principal vía de entrada al ciclo de cultivo fue derivada de los alimentos con $142 \mathrm{~kg} \mathrm{ha}^{-1}$ año ${ }^{-1}$ (91\%); seguido del agua de los influentes con $14 \mathrm{~kg}$ $\mathrm{ha}^{-1}$ año-1 $(9 \%)$. Por otra parte, la salida de fósforo del sistema incluye la cosecha con el $74 \%$ del fósforo en forma de la biomasa de camarón. A través de los efluentes se intercambiaron el $26 \%$ del total de salidas, esto significa que el biofiltro recibió $42 \mathrm{~kg} \mathrm{ha}^{-1}$ año ${ }^{-1}$ en forma de $\mathrm{PO}_{4}^{-3}$. El fósforo acumulado en los sedimentos de los estanques constituyó en promedio $6 \mathrm{~kg} \mathrm{ha}^{-1}$ año-1 (Tabla 2). De

Tabla 1. Calidad de agua del influente, efluente, zona marina y humedales de manglar (promedio \pm error estándar) / Water quality monitoring for the influent pond, effluent pond, sea zone and mangrove swamp (mean \pm standard error)

\begin{tabular}{|c|c|c|c|c|c|c|}
\hline Variable & Influente & Efluente & $\begin{array}{c}\text { Zona } \\
\text { Marina }\end{array}$ & Ciénaga & $\mathrm{H}$ & Probabilidad \\
\hline $\mathrm{NO}_{2}^{-}+\mathrm{NO}_{3}^{-}\left(\mathrm{mg} \mathrm{l}^{-1}\right)$ & ${ }^{\mathrm{a}} 4,69 \pm 0,26$ & ${ }^{b} 0,56 \pm 0,20$ & ${ }^{\mathrm{b}} 0,44 \pm 0,14$ & ${ }^{\mathrm{b}} 0,28 \pm 0,09$ & 14,69 & $0,002^{*}$ \\
\hline $\mathrm{NH}_{4}^{+}\left(\mathrm{mg} \mathrm{l}^{-1}\right)$ & ${ }^{b} 0,11 \pm 0,01$ & ${ }^{\mathrm{a}} 0,56 \pm 0,49$ & ${ }^{b} 0,05 \pm 0,03$ & ${ }^{\mathrm{a}} 0,32 \pm 0,12$ & 13,47 & $0,003^{*}$ \\
\hline $\mathrm{NID}\left(\mathrm{mg} \mathrm{l}^{-1}\right)$ & ${ }^{a} 4,83 \pm 0,26$ & ${ }^{b} 1,34 \pm 0,60$ & ${ }^{b} 0,54 \pm 0,13$ & ${ }^{\mathrm{b}} 0,55 \pm 0,15$ & 13,74 & $0,003^{*}$ \\
\hline $\mathrm{PO}_{4}^{-3}\left(\mathrm{mg} \mathrm{l}^{-1}\right)$ & ${ }^{\mathrm{b}} 0,06 \pm 0,01$ & ${ }^{\mathrm{a}} 0,18 \pm 0,03$ & ${ }^{\mathrm{b}} 0,09 \pm 0,02$ & ${ }^{\mathrm{b}} 0,09 \pm 0,02$ & 11,81 & $0,008^{*}$ \\
\hline Temperatura $\left({ }^{\circ} \mathrm{C}\right)$ & $26,6 \pm 0,79$ & $27,1 \pm 0,59$ & $27,6 \pm 0,5$ & $25,3 \pm 4,81$ & 0,540 & 0,910 \\
\hline Salinidad (ups) & ${ }^{b} 19,4 \pm 0,46$ & ${ }^{b} 24,0 \pm 0,22$ & ${ }^{a} 35,6 \pm 0,67$ & ${ }^{a} 36,5 \pm 0,41$ & 14,27 & $0,002 *$ \\
\hline Oxígeno disuelto $\left(\mathrm{mg} \mathrm{l}^{-1}\right)$ & $5,81 \pm 1,12$ & $5,88 \pm 0,54$ & $5,22 \pm 1,65$ & $5,11 \pm 1,00$ & 2,15 & 0,542 \\
\hline $\operatorname{TSS}\left(\mathrm{mg} \mathrm{l}^{-1}\right)$ & $b_{31,6} \pm 2,45$ & ${ }^{\mathrm{a}} 163,4 \pm 10,1$ & ${ }^{\mathrm{a}} 167,9 \pm 13,8$ & b $88,2 \pm 10,8$ & 16,90 & $0,001^{*}$ \\
\hline $\operatorname{VSS}\left(\mathrm{mg} \mathrm{l}^{-1}\right)$ & $\mathrm{b}_{5,67} \pm 1,35$ & ${ }^{\mathrm{a}} 52,0 \pm 6,83$ & ${ }^{c} 33,4 \pm 5,92$ & ${ }^{\mathrm{b}} 12,0 \pm 10,5$ & 16,09 & $0,001 *$ \\
\hline Clorofila-a $\left(\mu \mathrm{g} \mathrm{l}^{-1}\right)$ & ${ }^{\mathrm{c}} 1,84 \pm 0,19$ & ${ }^{\mathrm{a}} 157,1 \pm 23,7$ & b $4,67 \pm 5,19$ & ${ }^{\mathrm{b}} 5,99 \pm 0,62$ & 13,53 & $0,003^{*}$ \\
\hline
\end{tabular}

Nivel de significancia señalado con $*$ indica diferencias significativas $(P<0,01)$. Letras distintas, los grupos que mostraron diferencias $P<0,05$ 
Tabla 2. Intercambio y balance de masas de nitrógeno y fósforo $\left(\mathrm{kg} \mathrm{ha}^{-1} \mathrm{año}^{-1}\right)$ en los estanques de cultivo (Est) en 3 ciclos de producción / Exchange and nitrogen and phosphate budget ( $\mathrm{kg}$ $\mathrm{ha}^{-1} \mathrm{yr}^{-1}$ ) for shrimp pond (Est) during 3 growout cycle

\begin{tabular}{|c|c|c|c|c|c|c|c|c|}
\hline \multirow{2}{*}{ Ciclo } & \multicolumn{4}{|c|}{ Balance $\mathrm{N}\left(\mathrm{kg} \mathrm{ha}^{-1} \mathrm{año}^{-1}\right)$} & \multicolumn{4}{|c|}{ Balance $P\left(\mathrm{~kg} \mathrm{ha}^{-1} \mathrm{año}^{-1}\right)$} \\
\hline & Est 1 & Est 2 & Est 3 & Promedio & Est 1 & Est 2 & Est 3 & Promedio \\
\hline \multicolumn{9}{|l|}{ Entrada } \\
\hline Alimento & 506 & 692 & 638 & 612 & 118 & 161 & 148 & 142 \\
\hline Influente & 746 & 1.233 & 971 & 983 & 11 & 18 & 13 & 14 \\
\hline Fertilizante & 0,84 & 1,41 & 1,41 & 1,22 & n.r. & n.r. & n.r. & n.r. \\
\hline Siembra & 1,27 & 1,43 & 1,30 & 1,34 & 0,14 & 0,18 & 0,16 & 0,16 \\
\hline Total & 1.255 & 1.928 & 1.612 & 1.598 & 129 & 179 & 162 & 157 \\
\hline \multicolumn{9}{|l|}{ Salida } \\
\hline Cosecha & 866 & 1.164 & 1.072 & 1.034 & 100 & 134 & 114 & 116 \\
\hline Efluentes & 249 & 545 & 282 & 359 & 27 & 44 & 54 & 42 \\
\hline Sedimentos & 47 & 82 & 70 & 67 & 4 & 7 & 6 & 6 \\
\hline Remanente & 92 & 136 & 187 & 138 & -2 & -6 & -12 & -7 \\
\hline Total & 1.255 & 1.928 & 1.612 & 1.598 & 129 & 179 & 162 & 157 \\
\hline
\end{tabular}

n.r.= no registrado

acuerdo al balance de las entradas y salidas del fósforo, se determinó que la pérdida total neta promedio fue de 7 $\mathrm{kg} \mathrm{ha}^{-1}$ año ${ }^{-1}(4,2 \%)$ a través de vías no cuantificadas.

\section{EFICIENCIA EN LA REDUCCIÓN DE NUTRIENTES POR EL BIOFILTRO}

Respecto al análisis de las concentraciones de nutrientes, sólidos suspendidos y características fisicoquímicas del agua entre los efluentes, el biofiltro y la descarga del biofiltro no presentaron diferencias estadísticas $(P>0,03)$, a excepción del $\mathrm{PO}_{4}^{-3}$, (Tabla 3). La concentración de $\mathrm{PO}_{4}^{-3}$ al entrar al biofiltro fue de $0,18 \mathrm{mg} \mathrm{l}^{-1}$ y $0,08 \mathrm{mg} \mathrm{l}^{-1}$ en la salida. Respecto $\mathrm{NO}_{2}^{-}+\mathrm{NO}_{3}^{-}$, las condiciones del biofiltro favoreció su incremento, ya que las concentración en la entrada fue de 0,70 y $0,99 \mathrm{mg} \mathrm{l}^{-1}$ en la salida. La mayor concentración de $\mathrm{NH}_{4}^{+}$se registró en la columna de agua dentro del biofiltro con 1,46 $\mathrm{mg} \mathrm{l}^{-1}$ a diferencia de la entrada y salida que fue menor a $0,93 \mathrm{mg} \mathrm{l}^{-1}$. El registro de SST fue mayor con 165,5 $\mathrm{mg} \mathrm{l}^{-1}$ en los efluentes de entrada al biofiltro, pero luego decrece a 136,6 $\mathrm{mg} \mathrm{l}^{-1}$ en la salida (Tabla 3). Los SSV no presentaron cambios entre los efluentes y el biofiltro, los valores registrados fueron 52,7 y 48,0 $\mathrm{mg} \mathrm{l}^{-1}$, respectivamente. Dentro del biofiltro se presentó incremento en la comunidad fitoplanctónica, ya que la concentración de clorofila-a fue de 279,6 $\mu \mathrm{g} \mathrm{l}^{-1}$; a pesar de este incremento, esta no fue exportada a la zona marina, ya que la concentración en la descarga del biofiltro fue de 131,7 $\mu \mathrm{g} \mathrm{l}^{-1}$ (Tabla 3).
Respecto al flujo promedio diario del agua del efluente hacia el biofiltro fue de $924 \mathrm{~m}^{3} \mathrm{~d}^{-1} \mathrm{y}$ del biofiltro a la zona marina fue en promedio diario de $400 \mathrm{~m}^{3} \mathrm{~d}^{-1}$, la diferencias entre el flujo de entrada y de salida corresponde a los efluentes almacenados en el biofiltro. Con estos valores de flujo fue posible estimar la carga de materiales antes y después de pasar por el biofiltro. Cuando se analizaron los materiales del efluente a través de las cargas, los resultados fueron diferentes al de las concentraciones. En todos los casos se presentaron diferentes grados de reducción entre la entrada y salida del biofiltro (Tabla 4). El $\mathrm{PO}_{4}^{-3}$ fue la variable que presentó mayor eficiencia en la retención. La carga promedio de $\mathrm{PO}_{4}^{-3}$ en la entrada fue de $0,17 \mathrm{~kg}$ día $^{-1}$ y de $0,03 \mathrm{~kg}_{\text {día }}{ }^{-1}$ en la salida del biofiltro. El biofiltro fue eficiente en la reducción de la carga de SST, SSV y la clorofila-a con el 68, 61 y $66 \%$ de eficiencia, respectivamente $(P \leq 0,01)$.

En el caso del nitrógeno, las eficiencias para cada una de las formas inorgánicas fue de $44 \%$ para el $\mathrm{NO}_{2}{ }^{-}+\mathrm{NO}_{3}{ }^{-}$y de $55 \%$ para el $\mathrm{NH}_{4}^{+}$(Tabla 4). El intervalo de la carga de $\mathrm{NO}_{2}{ }^{-}+\mathrm{NO}_{3}{ }^{-}$de los efluentes antes del biofiltro fue de 0,25 a 1,35 kg día ${ }^{-1}$ y de 0,64 a $0,19 \mathrm{~kg} \mathrm{día}^{-1}$ en la salida. Se determinó una reducción de la carga del $\mathrm{NH}_{4}^{+}$en donde la entrada fue 0,12 a 3,05 kg día ${ }^{-1}$ y de 0,09 a 0,58 kg día ${ }^{-1}$ en la salida del biofiltro (Tabla 4). Independientemente de la carga de entrada, el biofiltro funcionó reduciendo los nutrientes y los materiales en los efluentes. 
Tabla 3. Características de la calidad de agua (promedio \pm error estándar para cada variable) de los efluentes, biofiltro y descarga del biofiltro / Water quality characteristics (mean \pm standard error for each variable) of effluent pond, biofilter and biofilter discharge

\begin{tabular}{lrrr}
\hline Variable & Efluentes & \multicolumn{1}{c}{ Biofiltro } & Descarga del biofiltro \\
\hline $\mathrm{NO}_{2}{ }^{-}+\mathrm{NO}_{3}{ }^{-}\left(\mathrm{mg} \mathrm{l}^{-1}\right)$ & $0,70 \pm 0,20$ & $0,65 \pm 0,20$ & $0,99 \pm 0,23$ \\
$\mathrm{NH}_{4}{ }^{+}\left(\mathrm{mg} \mathrm{l}^{-1}\right)$ & $0,93 \pm 0,49$ & $1,45 \pm 0,52$ & $0,96 \pm 0,19$ \\
$\mathrm{PO}_{4}^{-3}\left(\mathrm{mg} \mathrm{l}^{-1}\right)^{*}$ & $0,18 \pm 0,03$ & $0,20 \pm 0,04$ & $0,08 \pm 0,02$ \\
${\mathrm{Temperatura}\left({ }^{\circ} \mathrm{C}\right)}_{\mathrm{Salinidad}}^{26,52 \pm 0,78}$ & $27,96 \pm 0,79$ & $27,98 \pm 0,84$ \\
$\mathrm{SST}\left(\mathrm{mg} \mathrm{l}^{-1}\right)$ & $24,33 \pm 0,59$ & $24,35 \pm 0,70$ & $24,53 \pm 0,58$ \\
$\mathrm{SSV}\left(\mathrm{mg} \mathrm{l}^{-1}\right)$ & $165,5 \pm 14,1$ & $137,9 \pm 21,1$ & $136,6 \pm 30,8$ \\
Clorofila-a $\left(\mu \mathrm{g} \mathrm{l}^{-1}\right)$ & $52,8 \pm 6,83$ & $52,7 \pm 9,19$ & $48,03 \pm 15,7$ \\
\hline
\end{tabular}

*: variable que difiere significativamente entre grupos $(\mathrm{H}=6,44$ y $P<0,03)$

Tabla 4. Características y reducción de la carga $\left(\mathrm{kg} \mathrm{día}^{-1}\right)$ de nutrientes y sólidos suspendidos en los efluentes antes y después del biofiltro de manglar / Characteristics and loading rate ( $\left.\mathrm{kg} \mathrm{day}^{-1}\right)$ reduction of nutrients and suspended solids in effluent water before and after of the mangrove biofilter

\begin{tabular}{|c|c|c|c|c|c|c|c|}
\hline Variable & $\begin{array}{c}\mathrm{NO}_{2}^{-}+\mathrm{NO}_{3}^{-} \\
\left(\mathrm{kg} \mathrm{dia}^{-1}\right)\end{array}$ & $\begin{array}{c}\mathrm{NH}_{4}^{+} \\
\left(\mathrm{kg} \mathrm{día}^{-1}\right)\end{array}$ & $\begin{array}{c}\text { NID } \\
\left(\mathrm{kg} \mathrm{día}^{-1}\right)\end{array}$ & $\begin{array}{c}\mathrm{PO}_{4}^{-3} \\
\left(\mathrm{~kg} \mathrm{dia}^{-1}\right)\end{array}$ & $\begin{array}{c}\text { SST } \\
\left(\mathrm{kg} \mathrm{día}^{-1}\right)\end{array}$ & $\begin{array}{c}\text { SSV } \\
\left(\mathrm{kg} \mathrm{dia}^{-1}\right)\end{array}$ & $\begin{array}{c}\text { Clorofila- a } \\
\left(\mathrm{kg} \mathrm{día}^{-1}\right)\end{array}$ \\
\hline \multicolumn{8}{|l|}{ Antes del biofiltro } \\
\hline Promedio & 0,66 & 0,86 & 1,52 & 0,17 & 153,1 & 48,9 & 0,16 \\
\hline Error estándar & 0,44 & 1,10 & 1,35 & 0,06 & 32,1 & 15,5 & 0,05 \\
\hline Valor mínimo & 0,25 & 0,12 & 0,38 & 0,08 & 113,7 & 26,4 & 0,10 \\
\hline Valor máximo & 1,35 & 3,05 & 4,06 & 0,27 & 190,3 & 74,0 & 0,26 \\
\hline \multicolumn{8}{|l|}{ Después del biofiltro } \\
\hline Promedio & 0,37 & 0,38 & 0,76 & 0,03 & 49,1 & 19,2 & 0,05 \\
\hline Error estándar & 0,17 & 0,19 & 0,32 & 0,02 & 19,7 & 15,4 & 0,03 \\
\hline Valor mínimo & 0,19 & 0,09 & 0,38 & 0,01 & 29,6 & 9,3 & 0,00 \\
\hline Valor máximo & 0,64 & 0,58 & 1,21 & 0,05 & 74,0 & 50,3 & 0,09 \\
\hline Reducción carga (\%) & 44 & 55 & 50 & 80 & 68 & 61 & 66 \\
\hline $\mathrm{H}$ & 1,3 & 0,4 & 0,6 & 8,3 & 8,3 & 5,8 & 8,3 \\
\hline$P \leq 0,01$ & 0,30 & 0,58 & 0,48 & $0,002^{*}$ & $0,002^{*}$ & $0,010^{*}$ & $0,002^{*}$ \\
\hline
\end{tabular}

*: indica diferencias significativas $(P<0,01)$ en las variables de calidad de agua entre antes y después de pasar el biofiltro

\section{Discusión}

La concentración de nitrógeno fue mayor en el influente (4,69 $\left.\mathrm{mg} \mathrm{l}^{-1}\right)$, debido a que el aporte fue principalmente en forma de $\mathrm{NO}_{2}^{-}+\mathrm{NO}_{3}^{-}$. La salinidad del influente para los estanques fue resultado de la mezcla de agua subterránea salobre con salinidad de 19,4 y de agua de mar con salinidad de 35,6. El agua de las descargas subterráneas en Yucatán se caracterizan por una alta concentración de $\mathrm{NO}_{2}{ }^{-}+\mathrm{NO}_{3}{ }^{-}$(Pérez-Ceballos \& Pacheco-Ávila 2004); baja salinidad (<5 ups), $\mathrm{NH}_{4}^{+}\left(0,29 \mathrm{mg} \mathrm{l}^{-1}\right)$ y $\mathrm{PO}_{4}^{-3}\left(0,07 \mathrm{mg} \mathrm{l}^{-1}\right)$; estos resultados están en concordancia con los obtenidos por Herrera-Silveira et al. (1998) y Aranda-Cirerol et al. (2006).

Se determinó que la principal entrada de nitrógeno al sistema de cultivo fue a través del agua del influente con $983 \mathrm{~kg} \mathrm{ha}^{-1} \mathrm{año}^{-1}$ y los alimentos para el caso del fósforo con $142 \mathrm{~kg} \mathrm{ha}^{-1}$ año-1 (91\%). Los alimentos serían la vía de entrada del $80 \%$ del nitrógeno y fósforo (Robertson \& Phillips 1995, Rivera-Monroy et al. 1999, Páez-Osuna 2001, 
Casillas-Hernandez et al. 2006). Los efluentes fueron la segunda vía en importancia para las salidas de nitrógeno y fósforo. En este sentido, este estudio determinó que para el caso del nitrógeno los estanques exportaron a través de sus efluentes $359 \mathrm{~kg} \mathrm{ha}^{-1}$ año-1 de NID principalmente como amonio y para el $\mathrm{PO}_{4}^{-3}$ fue de $42 \mathrm{~kg} \mathrm{ha}^{-1}$ año ${ }^{-1}$; a pesar de ello los manglares fueron capaces de reducir la carga de nutrientes y materiales de los efluentes de camaroneras y que tenían el destino final hacia la zona marina del norte de Yucatán.

La eficiencia del biofiltro usando manglares fue alta para el tratamiento de efluentes comparado con otros estudios utilizando manglares para filtrar efluentes (Gautier et al. 2001, Primavera et al. 2007). El biofiltro presentó la capacidad de retener el $44 \%$ del $\mathrm{NO}_{2}{ }^{-}+\mathrm{NO}_{3}{ }^{-}$, el $55 \%$ del $\mathrm{NH}_{4}^{+}$y el $80 \%$ del $\mathrm{PO}_{4}^{-3}$ de las descargas de nutrientes y sólidos suspendidos de la granja camaronera. Si se comparan las eficiencias en la retención de materiales por el biofiltro en este estudio con otros tratamientos como son las cuencas de sedimentación, estudios demostraron que retienen el 31\% de nitrógeno y $64 \%$ para el fósforo (Boyd 1990, Teichert-Coddington et al. 1999, Jones et al. 2001), esto sugiere que un sistema integrado entre manglar y laguna resulta ser mejor eficiente para el tratamiento de los efluentes. Otro estudio realizado, pero en humedales artificiales de pastizal salobre para el tratamiento de efluentes de camaroneras en Texas demostró eficiencias de 31\% para el fósforo y $65 \%$ para los SST y para el caso del nitrógeno el humedal funcionó modulando las concentraciones de $\mathrm{NH}_{4}^{+}$por debajo de los $2,0 \mathrm{mg} \mathrm{l}^{-1}$ y 0,4 $\mathrm{mg} \mathrm{l}^{-1}$ para el $\mathrm{NO}_{3}^{-}$(Tilley et al. 2002).

Las altas eficiencias de retención de fósforo pueden ser explicadas a través de la adsorción y precipitación del fósforo en el suelo cárstico de Yucatán. Es reconocido que los suelos de los humedales de manglar son considerados el mayor reservorio de fósforo (Alongi 1996, Mitsch \& Gosselink 1993). Por otra parte, la condición cárstica determina que el fósforo sea un recurso limitante para los manglares de Yucatán (Feller 1995). Bajo esta condición los manglares podrían estar favorecidos por la fertilización de fosforo a través de los efluentes. Por otra parte, la eficiencia en la sedimentación en el biofiltro fue de $68 \%$ del total de entrada de SST (153 kg día ${ }^{-1}$ ), la carga de sólidos suspendidos también podría tener implicaciones en la retención de fósforo. En sistemas de cultivo intensivos el fitoplancton es la principal fuente de sólidos suspendidos, ya que el enriquecimiento de nutrientes y el tiempo de residencia del agua favorecen los florecimientos algales que son parte esencial de los ciclos de cultivo acuáticos (Trott \& Alongi 2000).
La carga de sólidos suspendidos y clorofila-a presentaron eficiencias de remoción del biofiltro mayores a $61 \%$, siendo menor comparado con un estudio similar en Colombia donde se registraron eficiencias de retención del 95\%. Rivera-Monroy et al. (1999) determinaron que los humedales de manglar pueden transformar a través de la desnitrificación de $1,49 \mathrm{~kg} \mathrm{~N} \mathrm{ha}^{-1}$ día $^{-1}$. Considerando la carga de NID en los efluentes a la entrada del biofiltro que fueron entre 0,38 y 4,06 kg día-1 podría potencialmente requerirse de 0,25 y 2,72 ha de manglar para retenerse el $100 \%$ vía desnitrificación de la descarga diaria de nitrógeno de los efluentes en esta granja intensiva. Estudios en Indonesia han demostrado que por cada estanque de cultivo se requieren entre 1,8-2,7 ha y 3,6-5,4 ha de manglar para depurar las carga de nitrógeno de los efluente en granjas semi-intensivas e intensivas, respectivamente (Primavera et al. 2007).

Para promover las tasas de nitrificación y desnitrificación en los manglares que reciben efluentes es recomendable el control del hidroperíodo (nivel, frecuencia y tiempo de inundación), dirigido hacia periodos de inundación y de sequía, ya que la aerobiosis (sequía) es mejor ambiente que las anaerobiosis (inundación) para los procesos de transformación de los nutrientes, con el manejo del hidroperíodo se incrementaría la eficiencia en la remoción del nitrógeno por medio de los procesos de nitrificacióndesnitrificación (Holguin et al. 2001, Leonard et al. 2003, Tam et al. 2009).

El tiempo de residencia del agua y la relación estanque/ área de humedal son factores importantes de considerar para el adecuado tratamiento de agua en los humedales de manglar. Por ejemplo, en humedales artificiales se ha observado que para mejorar la eficiencia en el reciclamiento de nutrientes de un humedal debe considerase mayores tiempos de residencia del agua mientras menor sea el tamaño del humedal (Tilley et al. 2002).

El impacto en el largo plazo de las descargas de efluentes en los manglares no es claro. Algunos estudios han demostrados que la fertilización estimula la productividad (Boto \& Wellington 1983); otros no han observado diferencias en cuanto a la concentración de nutrientes, hojas y la estructura de la vegetación (Wong et al. 1997). Por otra parte, Vaiphasa et al. (2007) reportaron que el exceso de sedimentos en los efluentes reduce la tasa de crecimiento e incrementa la mortalidad de algunas especies de manglar; por último, el exceso de nutrientes induce a la acumulación de nutrientes en las estructuras aéreas del manglar y no en la raíces, dando como resultado 
mayor susceptibilidad a déficit hídricos (Lovelock et al. 2009). Los impactos de la descargas varían de un manglar a otro, por eso no es posible predecir el impacto de largo plazo, ya que ello depende de las propias características del humedal de manglar dentro de un escenario ambiental y distribución de especies.

La definición del área del manglar que se requiere para procesar efluentes de camaroneras es criterio contundente para el manejo del biofiltro. Este conocimiento traería beneficios en la conservación de los manglares y sustento para implementar estrategias de restauración ecológica en zona aledañas a las camaroneras y a la recuperación de áreas de manglar en estanques abandonados. Las eficiencias de reducción determinadas en este estudio demostraron que los manglares son buenos reciclando el fósforo de los efluentes de camaroneras, pero son menos eficientes para retener el nitrógeno. También fue demostrada la magnitud de la fertilización y del potencial de contaminación de las aguas receptoras de los efluentes cuando no se emplea un sistema de tratamiento o no se mejora y cambia el manejo tradicional de los efluentes. El uso de los biofíltros de manglar podría resultar en una potencial estrategia para mitigar los efectos de contaminación por efluentes de camaroneras sobre la calidad de agua costera.

\section{Agradecimientos}

Los autores agradecen por la asistencia a miembros del laboratorio de Producción Primaria del CINVESTAV Unidad Mérida y en especial a la Q.I. Elsy Alvarado e M.C. Ileana Osorio. También agradecemos al Dr. Victor Rivera-Monroy (LSU) por sus recomendaciones en el diseño de trabajo. Este trabajo fue financiado con fondos del CINVESTAV, CONACyT y la granja camaronera Industrias Pecis S.A de C.V.

\section{LITERATURA CITADA}

Adame MF, V Virdis \& K Lovelock. 2010. Effect of geomorphological setting and rainfall on nutrient exchange in mangroves during tidal inundation. Marine and Freshwater Research 61: 1197-1206.

Alongi DM. 1996. The dynamic of benthic nutrient pool and fluxes in tropical mangrove forests. Journal of Marine Research 54: 123-148.

Aranda-Cirerol N, JA Herrera-Silveira \& F Comín. 2006. Nutrient water quality in a tropical coastal zone with groundwater discharge, northwest Yucatan, Mexico. Estuarine, Coastal and Shelf Science 68: 445-454.
Boto KG \& JT Wellington. 1983. Phosphorus and nitrogen nutritional status of a northern Australian mangrove forest. Marine Ecology Progress Series 11: 63-69.

Boyd CE. 1990. Water quality in ponds for aquaculture, 482 pp. Auburn University, Alabama.

Casillas-Hernandez R, F Magallon-Barajas, G PortilloClarck \& F Páez-Osuna. 2006. Nutrient mass balances in semi-intensive shrimp ponds from Sonora, Mexico using two feeding strategies: trays and mechanical dispersal. Aquaculture 258: 289-298.

CNA. 2010. Servicio Meteorológico Nacional, México. [en línea] <http://smn.cna.gob.mx/climatologia/normales/estacion/yuc/ NORMAL31029.TXT>

CONABIO. 2008. Consejo nacional para el conocimiento y uso de la biodiversidad. 2008. Manglares de México, 35 pp. Comisión Nacional para el Conocimiento y Uso de la Biodiversidad, México.

Feller IC. 1995. Effects of nutrient enrichment on growth and herbivory of dwarf red mangrove (Rhizophora mangle). Ecological Monographs 54: 477-505.

Feller IC \& A Chamberlain. 2007. Herbivore responses to nutrient enrichment and landscape heterogeneity in a mangrove ecosystem. Oecologia 153: 607-616.

Gautier D, J Amador \& F Newmark. 2001. The use of mangrove wetland as a biofilter to treat shrimp pond effluents: preliminary results of an experiment on the Caribbean coast of Colombia. Aquaculture Research 32: 787-799.

Herrera-Silveira JA \& J Ramirez. 1997. Salinity and nutrients in the coastal lagoons of Yucatan, Mexico. Verhein International Verein Limnologie 23: 1348-1351.

Herrera-Silveira JA, J Ramírez \& A Zaldívar-Jiménez. 1998. Overview and characterization of the hydrology and primary producers communities of selected coastal lagoons of Yucatan, Mexico. Aquatic Ecosystem Health and Management 1: 353-372.

Holguin G, P Vazquez \& Y Bashan. 2001. The role of sediment microorganisms in the productivity, conservation and rehabilitation of mangrove ecosystems: an overview. Biology and Fertility of Soils 33: 265-278.

Jones AB, MJ O’Donohuea, J Udy \& WC Dennison. 2001. Assessing ecological impacts of shrimp and sewage effluent: biological indicators with standard water quality analyses. Estuarine, Coastal and Shelf Science 52: 91-109.

Kadlec RH \& RL Knight. 1996. Treatment wetlands, 893 pp. Lewis Publishers, Boca Raton.

Kruskal WH \& WA Wallis. 1952. Use of ranks on one-criterion variance analysis. Journal of the American Statistical Association 47: 583-621. 
Leonard KM, SP Key \& R Srikanthan. 2003. A comparison of nitrification performance in gravity-flow and reciprocating constructed wetlands. In: Brebbia CA, D Almorza \& D Sales (eds). Water pollution, modelling, measuring and prediction, pp. 293-301. WIT Press, Southhampton.

Lovelock CE, MC Ball, KC Martin \& IC Feller. 2009. Nutrient enrichment increases mortality of mangroves. [on line] Plos One 4(5): e5600. <doi:10.1371/ journal.pone.0005600>.

Mitsch WJ \& JG Gosselink. 1993. Wetlands, 722 pp. Van Nostrand Reinhold, New York.

Morales-Ojeda S \& J Herrera-Silveira. 2010. Terrestrial and oceanic influence on spatial hydrochemistry and trophic status in subtropical marine near-shore waters. Water Research 44: 5949-5964.

Murphy J \& P Riley. 1962. A modified single solution method for determination of phosphate in natural waters. Analytica Chimica Acta 27: 31-36.

Nath S \& JP Bolte. 1998. A water budget model for pond aquaculture. Aquaculture Engineering 18: 175-188.

Nedwell DB. 1975. Inorganic nitrogen metabolism in a eutrophicated tropical mangrove estuary. Water Research 9: 221-231.

Olvera-Novoa MA. 2010. La acuacultura y su posible impacto en la biodiversidad. En: Durán R \& M Méndez (eds). Biodiversidad y desarrollo humano en Yucatán, pp. 116118. CICY, PPD-FMAM, CONABIO, SEDUMA, Mérida.

Páez-Osuna F. 2001. The environmental impact of shrimp aquaculture: causes, effects and mitigating alternatives. Environmental Management 28(1): 131-140.

Parsons TR, Y Maita \& CM Lalli. 1984. A manual of chemical and biological methods for seawater analysis, $173 \mathrm{pp}$. Pergamon Press, Oxford.

Pérez-Ceballos R \& J Pacheco-Ávila. 2004. Vulnerabilidad del agua subterránea a la contaminación de nitratos en el estado de Yucatán. Ingeniería 8(1): 33-42.

Primavera JH, JPAltamirano, HL Lebata, AA de los Reyes Jr. \& CL Pitogo. 2007. Mangrove and shrimp pond culture effluents in Aklan, Panais Is, Central Philippines. Bulletin of Marine Science 80(3): 795-804.

Quinn GP \& MJ Keough. 2002. Experimental design and analysis for biologist, 537 pp. Cambridge University Press, Cambridge.

Reyes RM. 2001. Hidrología y estado trófico de la columna de agua de la zona marina costera de Sisal. Tesis de Maestría, Instituto Tecnológico de Mérida, Yucatán, México, 123 pp.

Rivera-Monroy V \& R Twilley. 1996. The relative role of denitrification and immobilization in the fate of inorganic nitrogen in mangrove sediments (Terminos Lagoon, Mexico). Limnology and Oceanography 41(2): 284-296.
Rivera-Monroy VH, LA Torres, N Bahamon, F Newmark \& RR Twilley. 1999. The potential use of mangrove forests as nitrogen sinks of shrimp aquaculture pond effluents: the role of denitrification. Journal of the World Aquaculture Society 30: 12-25.

Robertson AI \& MJ Phillips. 1995. Mangrove as filters of shrimp pond effluent: predictions and biogeochemical research needs. Hydrobiologia 295: 311-321.

Sánchez-Carrillo S \& J Álvarez-Yépiz. 2008. Viabilidad de los manglares artificiales como sistemas de tratamiento de los efluentes camaronícolas en Latinoamérica. Revista Latinoamericana de Recursos Naturales 4(1): 17-30.

Solorzano L. 1969. Determination of ammonia in natural water by phenolhypochlorite method. Limnology and Oceanography 14: 799-801.

Statgraphics Centurion XVI. 2010. User manual, 297 pp. Statpoint Tecnologies, Inc. United State.

Strickland J \& T Parsons. 1972. A practical handbook of seawater analysis. Fisheries Research Board of Canada Bulletin, 169: 1-310.

Tam NF, AH Wong, MH Wong \& YS Wong. 2009. Mass balance of nitrogen in constructed mangrove wetlands receiving ammonium-rich wastewater: effects of tidal regime and carbon supply. Ecological Engineering 35: 453-462.

Teichert-Coddington DR, BD Rouse, A Potts \& CE Boyd. 1999. Treatment of harvest discharge from intensive shrimp ponds by settling. Aquaculture Engineering 19: 147-161.

Tilley DR, H Badrinarayanan, R Rosati \& J Son. 2002. Constructed wetlands as recirculation filters in large-scale shrimp aquaculture. Aquacultural Engineering 26: 81-109.

Trott LA \& DM Alongi. 2000. The impact of shrimp pond effluent on water quality and phytoplankton biomass in a tropical mangrove estuary. Marine Pollution Bulletin 40(11): 947-951.

Twilley RR \& V Rivera-Monroy. 2009. Ecogeomorphic models of nutrient biogeochemistry for mangrove wetlands. In: Perillo GM, E Wolanski, DR Cahoon \& MM Brinson (eds). Coastal wetlands: an integrated ecosystem approach, pp. 641-675. Elselvier, Amsterdam.

Vaiphasa WF, AK de Boer, S Skidmore, T Panitchart, T Vaiphasa, N Bamrongrugsa \& P Santitamnont. 2007. Impact of solid shrimp pond waste materials on mangrove growth and mortality: a case study from Pak Phanang, Thailand. Hydrobiologia 591: 47-57.

Wong YS, CY Lan, GZ Chen, SH Li, XR Chen, ZP Liu \& NF Tam. 1995. Effect of wastewater discharge on nutrient contamination of mangrove soils and plants. Hydrobiologia 295: 243-254.

Wong YS, NF Tam \& CY Lan. 1997. Mangrove wetlands as wastewater treatment facility: a field trial. Hydrobiologia 352: 49-59. 
Wu Y, A Chung, NF Tam, N Pi \& MH Wong. 2008.

Constructed Mangrove wetland as secondary treatment system for municipal wastewater. Ecological Engineering 34: 137-146.
Zaldívar-Jiménez A, JA Herrera-Silveira \& L Capurro. 2001. Soil salinity and community structure of two mangrove forests in Yucatan, southeastern Mexico. International Verhein International Verein Limnologie 27(3): 1707-1710.

Recibido el 4 de noviembre de 2011 y aceptado el 24 de septiembre de 2012

Editor Asociado: Gabriela Muñoz C. 